Document downloaded from:

http://hdl.handle.net/10251/57427

This paper must be cited as:

Cabanes Sempere, M.; Catalá Civera, JM.; Penaranda-Foix, FL.; Cozzo, C.; Vaucher, S.; Pouchon, MA. (2013). Characterization method of dielectric properties of free falling drops in a microwave processing cavity and its application in microwave internal gelation. Measurement Science and Technology. 24(9). doi:10.1088/0957-0233/24/9/095009.

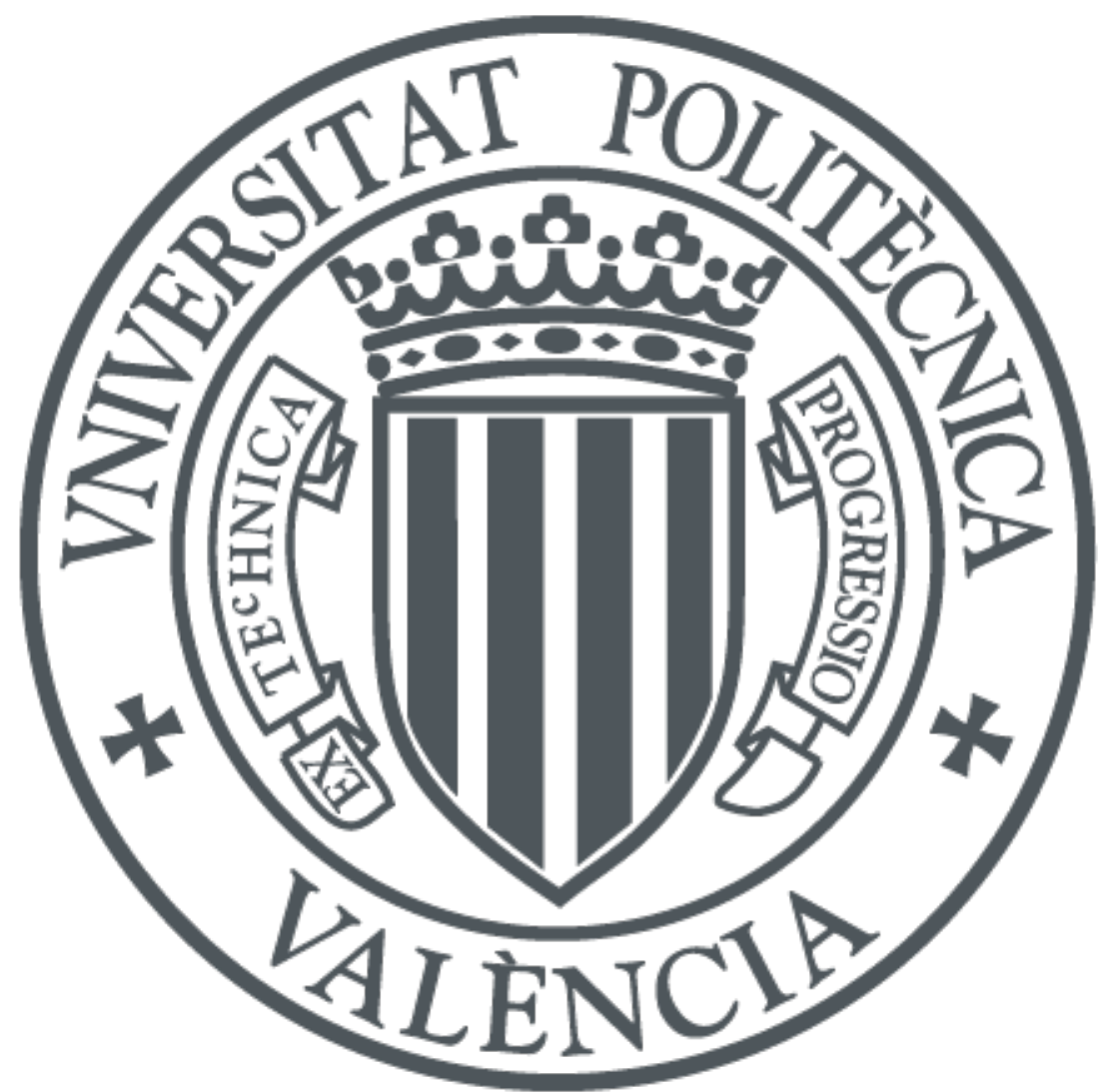

The final publication is available at

http://dx.doi.org/10.1088/0957-0233/24/9/095009

Copyright IOP Publishing: Hybrid Open Access

Additional Information 


\title{
1 Characterization method of dielectric properties of free 2 falling drops in a microwave processing cavity and its 3 application in Microwave Internal Gelation.
}

4

\author{
M Cabanes-Sempere ${ }^{\mathrm{a}, \mathrm{b}}$, J M Catalá-Civera ${ }^{\mathrm{b}}$, F L Peñaranda-Foix ${ }^{\mathrm{b}}$, \\ C Cozzo $^{\mathrm{a}}$, S Vaucher ${ }^{\mathrm{c}}$ and M A Pouchon ${ }^{\mathrm{a}}$ \\ ${ }^{\text {a }}$ Paul Scherrer Institut, 5232 Villigen PSI, Switzerland \\ ${ }^{\mathrm{b}}$ Universitat Politècnica de València, Camí de Vera s/n, 46022 València, Spain \\ c EMPA Fuerwerkstrasse 39, 3602 Thun, Switzerland \\ E-mail: maria.cabanes@psi.ch
}

\begin{abstract}
Microwave Internal Gelation (MIG) is a chemical process proposed for the production of nuclear particle fuel. The internal gelation reaction is triggered by a temperature increase of aqueous droplets falling by gravity by means of non-contact microwave heating. Due to the short residence time of a solution droplet in a microwave heating cavity, a detailed knowledge of the interaction between microwaves and chemical solution (shaped in small drops) is required. This paper describes a procedure that enables the measurement of dielectric properties of aqueous droplets that freely fall through a microwave cavity. These measurements provide the information to determine the optimal values of the parameters (such as frequency and power) that dictate the heating of such a material under microwaves.
\end{abstract}

Keywords: Cavity resonators, frequency response, dielectric measurements, perturbation method, Q-factor, nuclear fuels, fluid flow measurement

(Some figures may appear in colour only in the online journal)

\section{Introduction}

In the continuous aim to reduce the amount of minor actinides (MA) from the spent fuel of Light Water Reactors (LWR) and therefore reduce its radiotoxicity (radioactive toxicity), i.e. the hazard to humans from ingestion, inhalation and exposure to the radionuclides of spent fuel and High Level Waste (HLW) (see Figure 1.2 in [1]), new nuclear fuel concepts have been developed. Sphere-pac fuel (SP) offers a good possibility to reintroduce the MA in a fuel matrix and to burn them in a fast reactor [2], which facilitates a multi-cycle because of its breeding feature, or in a sub-critical fast system, i.e. an Accelerator Driven System (ADS), where its sub-criticality allows higher MA contents than a normal fast reactor reducing efficiently the radiotoxicity in one step. By this procedure, it is expected to cut down the necessary decay times of the nuclear waste, 100.000 years for MA to 1.000 years for fission products, before it reaches the radiotoxicity level of uranium.

In this concept, the fuel is in form of small spheres instead of the standard pellets. The particles (microspheres) are compacted into the pin by vibro-packing. Usually a bimodal size distribution is utilized. The smaller microspheres occupy the space between the large spheres resulting in a high fuel density [3]. With the lower production complexity, the SP fuel has important advantages; it especially avoids presses and grinding machines, used in the fabrication of pellets, 
decreasing the components maintenance requirements.

Nuclear fuel sphere particles are produced from a base solution, already containing all the elements, by internal gelation, which guarantees a good material homogeneity and a lower contamination risk compared to the classical pellet fabrication [4]. Internal gelation is an aqueous chemical reaction occurring when one of the precursors is heated up to $80 \pm 5^{\circ} \mathrm{C}$. Traditionally, the required heat to trigger the reaction is brought through conduction by contact of the sample with hot silicon oil. However this procedure induces an oil contamination of the fuel particles and requires a recycling step of the silicon oil and the organic solvents necessary to clean the particles from oil. In order to avoid contamination and consequently the recycling phase, an improvement of the system based on electromagnetic heating has been considered [5]-[8]. In the Microwave Internal Gelation (MIG) unit, the microwaves represent a much simpler and safer alternative: the contactless volumetric heating facilitates the remote production of the fuel in hot cells and furthermore reduces the contaminated liquid waste.

The use of microwaves for internal gelation has previously been reported in [5], [6] at frequencies in the S-band and in [4], [7], [8] in the X-band. However, the characterization of the feed solution droplets for the nuclear fuel production has not been made in any of these previous studies and consequently there is no reasonable election of the best frequency and power to be used in the production. In the MIG system, the heating time is very short (in the order of tens of milliseconds), therefore the microwave heating parameters have to be optimized and a good knowledge of the interaction between the microwaves and the samples must be achieved [9].

In this paper a new procedure that enables the measurement of dielectric properties of aqueous droplets that freely fall through a microwave cavity is described. First the justification of the selected frequency range and the need to develop a new measurement technique is presented. Then the guidelines to follow during the method are described. Finally, after the results the limitations and possibilities of the method are discussed.

\section{Thermal aspects of the Microwave Internal Gelation (MIG)}

Figure 1 shows the schematic of the MIG system developed to trigger the chemical reaction. The MIG unit consists of a microwave cavity where samples in the form of beads are heated during their free fall from a droplet generator at the top of the central axis [8]. The microwave cavity is designed here as a rectangular cavity working with $\mathrm{TE}_{10 \mathrm{~N}}$ modes. The drop generator provides samples with diameters from $0.4 \mathrm{~mm}$ to $3 \mathrm{~mm}$ by changing its nozzle size. Small drops are ejected by means of vibration and pressure whereas larger drops are simply detaching by gravity from the nozzle. A cooling liquid circulates in the walls of the droplet generator in order to prevent heating of the feed solution and the resulting premature solidification.

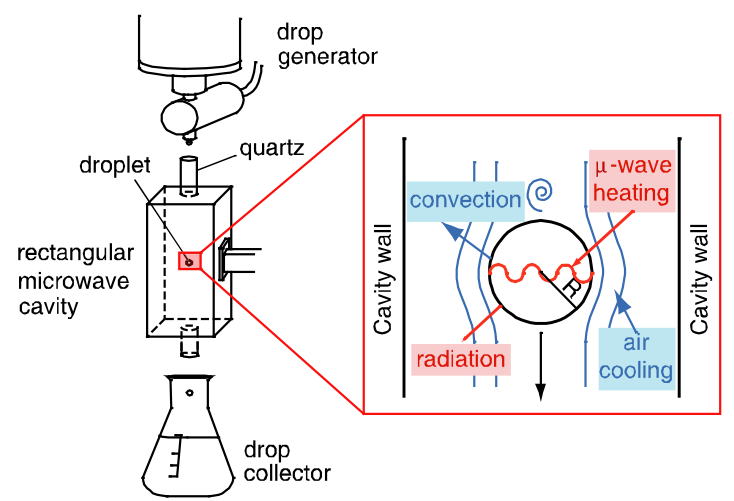

Figure 1. Schematic view of the MIG system. 
The residence time of the drops inside the cavity is very short (in the order of a few tens of milliseconds) and therefore the thermal behaviour of these drops, i.e. the increase of their temperature to trigger the gelation, depends on several parameters (Figure 1 right), such as the size of the droplet, thermal and dielectric properties of the solution and the frequency and power of the microwave signal.

\subsection{Microwave Heating of Droplets}

Depending on the frequency and on the dielectric properties of a material, the charge displacement produced by the applied electromagnetic field varies. The energy of microwaves is dissipated as heat in the samples and therefore the transfer satisfies the first law of thermodynamics. The Fourier energy balance equation [10], [11] allows the calculation of the temperature diffusion inside the material from the following equation:

where $\rho$ is material density in $\left(\mathrm{kg} \cdot \mathrm{m}^{-3}\right), c_{p}$ is specific heat in $\left(\mathrm{J} \cdot \mathrm{kg}^{-1} \cdot \mathrm{K}^{-1}\right), T$ is temperature in $(\mathrm{K}), t$ is time in seconds and $k_{T}$ is thermal conductivity in $\left(\mathrm{W} \cdot \mathrm{m}^{-1} \cdot \mathrm{K}^{-1}\right)$. The term on the left side of the equation represents the energy stored as heat, the first term on the right is the Fourier net heat input by conduction and $Q_{n e t}\left(\mathrm{~W} \cdot \mathrm{m}^{-3}\right)$ represents the net rate of heat generation in the material volume:

$$
Q_{\text {net }}=Q_{\text {gen }}+Q_{\text {loss }}+Q_{\text {local }}+Q_{\text {ph }}
$$

where $Q_{\text {net }}$ depends on the electromagnetic and thermal boundary conditions and can be solved by computing the electric field distribution within the sample in order to determine the microwave heat generation rate $\left(Q_{g e n}\right)$, the loss rate referred to the surface convection and radiation of the energy $\left(Q_{\text {loss }}\right)$, the heat rate generated by any local or chemical reaction $\left(Q_{\text {local }}\right)$ and that derivated from phase changes or material transformation $\left(Q_{p h}\right)$. $Q_{g e n}$ value is null in conventional heating, where the material is heated by contact at the surface according to the thermal boundary conditions. The microwave induced heating rate is given by [10]:

$$
Q_{g e n}=\omega \varepsilon_{0} \varepsilon^{\prime \prime}\left|E_{r m s}^{2}\right|
$$

where $\omega=2 \pi f_{0}$ is given by the resonant frequency $f_{0}(\mathrm{~Hz})$ of the cavity, $E_{r m s}$ is the electric field $\left(\mathrm{V} \cdot \mathrm{m}^{-1}\right)$ supplied to the cavity by an external generator, $\varepsilon_{0}$ is the vacuum permittivity $\left(\mathrm{F} \cdot \mathrm{m}^{-1}\right)$ and $\varepsilon^{\prime \prime}$ is the loss factor of the material (workload).

\subsection{FDTD Formulation}

To analyze the temperature evolution inside the drop during the heating process by microwaves, according to previous equations, a one-dimensional Finite Difference Time Domain (FDTD) model is applied. This model could be used in rectangular, cylindrical and spherical coordinates.

The small liquid drops used for the microwave internal gelation have a high surface tension and can be considered as spheres; therefore the spherical model is applied as: 
$2 \frac{\partial T}{\partial t}=\left(\frac{k_{T}}{\rho c_{P}}\right) \frac{1}{r^{2}} \frac{\partial}{\partial r}\left(r^{2} \frac{\partial T}{\partial r}\right)+\left(\frac{1}{\rho c_{P}}\right) Q_{g e n}$

3

where $r$ is the radial dimension (m) and $t$ is the time (s).

Combining the energy equations (1), (3) and (4) and following the procedure presented in [12], based on the Crank-Nicholson strategy [13], the temperature profile in a droplet heated by microwaves can be obtained for each time step.

The model is adjusted to the real case through the boundary conditions and the heating generation rate $Q_{g e n}$, which is dependent on the features of the cavity design. Since the spheres are surrounded by air, Neumann boundary conditions are applied to consider the convection [12].



Figure 2. FDTD simulation of the heating rate for a water drop using either 12 or $2.45 \mathrm{GHz}$ (incident power $150 \mathrm{~W}$, drop diameter $2.2 \mathrm{~mm}$ ).

Figure 2 displays the temperature evolution of a water drop $\left(\rho=1000 \mathrm{~kg} \cdot \mathrm{m}^{-3}\right.$, $\left.c_{p}=4190 \mathrm{~J} \cdot \mathrm{kg}^{-1} \cdot \mathrm{K}^{-1}, k_{T}=0.6 \mathrm{~W} \cdot \mathrm{m}^{-1} \cdot \mathrm{K}^{-1}\right)$ during free fall from a drop generator $(t=0 \mathrm{~s})$ through a microwave rectangular cavity $(0.0869<t<0.2199 \mathrm{~s})$ as a function of residence time. Free convection of the air is considered as a main boundary condition $\left(h_{T}=250 \mathrm{~W} \cdot \mathrm{m}^{-2} \cdot \mathrm{K}^{-1}\right)$. The water drop has $2.2 \mathrm{~mm}$ diameter and takes $133 \mathrm{~ms}$ to cross the cavity, which length is approximately $200 \mathrm{~mm}$ and which top entrance is located $37 \mathrm{~mm}$ from the drop generator. The cavity, simulated and applied to the FDTD scheme described above through the $Q_{g e n}$ term, is working with the $\mathrm{TE}_{10 \mathrm{~N}}$ mode and fed with a microwave power of 150 watts.

Figure 2 shows the different temperature rates that can be achieved by designing the cavity at different frequencies. At X-band a water drop with an initial temperature of $25{ }^{\circ} \mathrm{C}$ reaches the internal gelation temperature $\left(80 \pm 5^{\circ} \mathrm{C}\right)$, which corroborates the experiments performed by other authors in [4], [7], [8]. However, over the microwave frequency range in the S-band at $2.45 \mathrm{GHz}$, the drop temperature hardly reaches $30^{\circ} \mathrm{C}$ from the same initial temperature. Preliminary studies at this frequency reported high power rates e.g. $5000 \mathrm{~W}$ [5] or pre-heating by other sources [6] to arrive at the minimum temperature increase needed to trigger the gelation.

The oscillations of the temperature evolution in Figure 2 correspond to the distribution of EM fields inside the microwave cavity that for $\mathrm{X}$-band was $\mathrm{TE}_{1,0,15}$ meanwhile for the S-band was $\mathrm{TE}_{101}$ for a total cavity length of approximately $200 \mathrm{~mm}$. Figure 3 shows the $Q_{g e n}$ profile related with the generated fields inside the cavity for both cases. 
Figure 3. $Q_{\text {gen }}$ profile $(0.0869<t<0.2199 \mathrm{~s})$ applied to the FDTD simulation of Fig. 3 (incident power $150 \mathrm{~W}$, drop diameter $2.2 \mathrm{~mm}$ ).

The FDTD model, as it has been shown for the case of water, can provide accurately the temperature diffusion according to the material properties for a selected frequency and power.

8 Therefore, the dielectric properties of the feed solution for the production of fuel are also required 9 in order to determine the frequency and power used for the MIG. On the other hand, the short 10 residence time inside the cavity complicates the measurement of these properties with a Vector which is described in the following section.

\section{Principle of the method}

Over this section the method for the dielectric properties evaluation of a material in form of drops is introduced. First the experimental setup for the measurements of the resonant frequency is described. Afterwards the characteristics of the feed solution (material of main importance within the project where this method has been developed) are explained. Finally the procedure of the method in order to obtain satisfactory results is presented.

\subsection{Experimental Setup}

Figure 4 represents a schematic view of the dielectric measurements setup. A microwave cavity of cylindrical section has been designed here operating in the mode $\mathrm{TM}_{010}$ around the frequency of $10 \mathrm{GHz}$ (50 mm length, $9 \mathrm{~mm}$ radius, $4.05 \mathrm{~mm}$ top and bottom holes radius). The length of the cavity has been designed considering two main principles: on one hand, the maximum length i.e. the longest residence time of the drop inside the cavity has been selected; on the other hand the operating resonant mode $\left(\mathrm{TM}_{010}\right)$ remains at the lowest frequency avoiding the confusion with high order modes in the same frequency band. The microwave cavity is connected, either through a magnetic loop or an aperture in a rectangular waveguide, to a Vector Network Analyzer (VNA). The magnetic loop was used in a previous study run with water drops in order to prove the method feasibility [14]. In the current work the use of an aperture enables the measurement of a liquid while changing its temperature. In this case the feed solution described in section 3.2 is refrigerated to avoid a premature gelation and this refrigeration is also applied to the cavity. 




Figure 4. Experimental setup for the measurement of dielectric properties of a single drop. Cavity: 50 mm length, 9 $\mathrm{mm}$ radius, $4.05 \mathrm{~mm}$ top and bottom holes radius. Drop $2.9 \mathrm{~mm}$ diameter. Temperature $25{ }^{\circ} \mathrm{C}$.

The mode $\mathrm{TM}_{010}$ has been selected for this application because of its maximum interaction with the spheres due to the invariant electric field pattern in height, concentrated along the axis of the cavity. Figure 5 displays the magnitude of the Electric Field distribution in the cavity determined with the aid of the 3D EM Simulator QW3D [15]. Ideally the $\mathrm{TM}_{010}$ mode should keep the electric field constant along the cavity height. However, the apertures at the top and the bottom of the cavity perturb the nominal electric field reducing the intensity at the borders and concentrating the maximum at the center of the cavity, where the interaction of microwaves with the drops will be more enhanced.

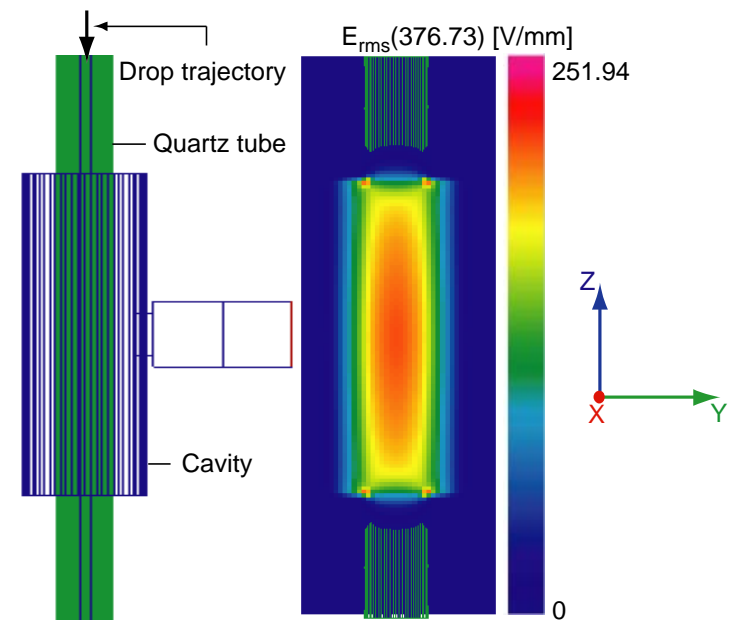

Figure 5. QW3D simulation (frontal and transversal view) of the magnitude of the E-Field distribution in a TM010 microwave cylindrical cavity at $10 \mathrm{GHz}$.

The solution falls from the drop generator through holes at the top and the bottom of the cavity. A quartz tube ( $4 \mathrm{~mm}$ outer radius $-2.9 \mathrm{~mm}$ inner radius) is placed inside the microwave cavity along the axis to protect the walls from contamination when the samples are passing through.

\subsection{Feed Solution}

For reasons of versatility in this early stage of development, the studied drops consist of an inactive metal surrogate. Cerium is chosen because of its physico-chemical similarity with actinide elements, especially Plutonium $(\mathrm{Pu})$ [16], [17]. The sample is a mixture of a metallic solution (ceric ammonium nitrate) and the hexamethylenetetramine (HMTA) solution (composed 
of HMTA, urea and water).

The HMTA decomposes with temperature increase and raises the $\mathrm{pH}$ of the solution, which causes the gelation. In order to prevent a premature gelation, the solution is cooled down to a temperature between $0{ }^{\circ} \mathrm{C}$ and $5{ }^{\circ} \mathrm{C}$ before the measurements. The main content of the drops is water, which is also used as a specimen for the first experiments reported in this work. The nozzle was adjusted to produce $1.47 \mathrm{~mm}$ radius water drops and $1.3 \mathrm{~mm}$ radius HMTA cerium based drops.

In the Sphere-pac concept two size fractions are used. The method described on this paper treats the coarse fraction.

\subsection{Drop Measurement}

The resonances measurement in microwave cavities with static samples can be carried out in a straightforward manner with an automatic vector network analyzer (VNA). However the conditions are different for a single particle in movement e.g. a drop falling by gravity. Because the residence time of a single drop crossing the cavity is very short e.g. $52 \mathrm{~ms}$ and much faster than the analyzing VNA device response ( 20 ms, 100 points, Rohde\&Schwarz ZVA 50), the frequency response cannot easily be captured. Therefore a new procedure is needed for this case.

The method for the free falling drop case consists of the excitation of the cylindrical cavity with a fixed frequency while the input return losses parameter $\left(\mathrm{S}_{11}\right.$ amplitude and phase) is recorded with the aid of a VNA. The fixed frequency is varied stepwise while the water drops are falling by gravity along the axis of the cavity. Measurements are repeated over a frequency range large enough to recover the $S_{11}$ response and evaluate the resonant frequency displacement with regard to the other cases (i.e. empty cavity that can be straightforward measured with the VNA).

Figure 6 shows the measurements of the cavity with droplets at a single frequency as a function of time. Each pulse represents one drop through the cavity. The registered pulse length corresponds to the time that the drop takes to cross the cylindrical cavity. In the current study the time is $52 \mathrm{~ms}$.



Figure 6. $\mathrm{S}_{11}$ measurement of the $\mathrm{TM}_{010}$ cylindrical microwave cavity at a frequency of $9.998 \mathrm{GHz}$ while 3 consecutive drops are passing through.

If the pulses are enlarged, as seen in Figure 7, it is possible to appreciate that the shape of the pulses changes depending on if the selected frequency is the resonance of the empty cavity, the resonance of the cavity with the drop or an intermediate step between both resonant frequencies. 



Figure 7. $\mathrm{S}_{11}$ measurement of the TM010 cylindrical microwave cavity for a single drop at: a) the resonant frequency of the empty cavity $10.054 \mathrm{GHz}, \mathrm{b}$ ) a transient frequency $10.023 \mathrm{GHz}$ between resonances, and c) the resonant frequency of the cavity with the drop $10.005 \mathrm{GHz}$.

At the resonant frequency of the empty cavity, $10.054 \mathrm{GHz}$ (Figure 7 a)), it is possible to observe how the $S_{11}$ response is detuned and therefore presents a minimum value during the time that the drop remains inside the cavity. Using this value as starting point the frequency is decreased until the complete resonant frequency of the cavity with the drop is recovered. At $10.023 \mathrm{GHz}$ (Figure $7 \mathrm{~b}$ )) the $\mathrm{S}_{11}$ parameter has a symmetrical response with respect to the middle point of the cavity. The return losses value is now reduced at the borders because this frequency is crossing the transient below the empty cavity resonant frequency. However this point is entering the transient above the resonant frequency of the cavity with a drop in the middle and therefore the value at the center is increased. At $10.005 \mathrm{GHz}$ (Figure 7 c)) the $\mathrm{S}_{11}$ parameter is maximal when the drop is at the center of the cavity i.e. this frequency corresponds to the resonant frequency of the cavity with the drop centered inside it.

The middle point of these pulses (marked in Figure 7 and 10 with a red triangle, square and circle for the $10.054 \mathrm{GHz}, 10.023 \mathrm{GHz}$ and $10.005 \mathrm{GHz}$ respectively), which corresponds to the case when the drop is at the cavity center, is the one elected to reconstruct the frequency response.

Figure 8 displays an overview of the $S_{11}$ amplitude measurements over time for all the frequency steps used in this drop measurement for the frequency band of the selected mode. It is possible to observe the transient of the pulse shape from the empty (highest frequency) to the 
1 drop center loaded cavity case as a function of frequency. Cutting the representation along the $240 \mathrm{~ms}$ point on the time axes also results in the full frequency response when the drop is in the 3 center of the cavity, i.e. in case of maximal deviation of frequency with respect the case of the 4 empty cavity (see approximation represented with a light green line).

\subsection{Drop Simulation}

12

The software CST microwave studio [18] is used with the main intention to reproduce the behavior of a drop while passing through the cavity. The cylindrical cavity used during the experiments is designed with the same characteristics than those described in 3.1. A drop is moved from $0 \mathrm{~mm}$ (cavity entrance) until $50 \mathrm{~mm}$ (cavity exit) in $1 \mathrm{~mm}$ steps. The final result is close to the one represented in Figure 8. In Figure 9 the input return losses are represented for positions taken every 5 steps.

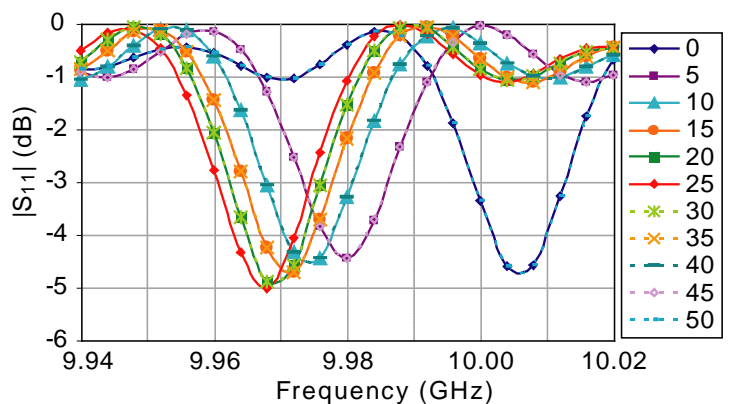

Figure 9. CST simulation of the resonant frequency shift while a $1.45 \mathrm{~mm}$ radius drop is passing through a cylindrical microwave cavity.

The frequency response is symmetrical with respect to the center of the cavity: for a droplet travelling from the top to the center of the cavity the resonant frequency is shifted from a higher 
of the resonances are repeated in the contrary direction, i.e. from a lower to a higher frequency.

This representation gives further information about the interaction of the drop with respect to the cavity. Most of the resonances are close in frequency $( \pm 10 \mathrm{MHz})$ as the drop is close to the center. Any frequency of this range, and not only the frequency of the maximal excursion (center of the cavity), can be set in the frequency generator for the heating of drops in a microwave cavity to provide a maximal interaction with the fields.

\section{Measurements and results}

Figure 10 left shows the Smith chart representation of the frequency response of the input return losses $\left(S_{11}\right)$ of the microwave cavity with different materials at its center reconstructed stepwise with the procedure mentioned above for the resonant mode $\mathrm{TM}_{010}$. The $\mathrm{S}_{11}$ is measured through an aperture in a rectangular waveguide (See Fig. 2 and Fig. 3 in [14] for the case of measurement through a magnetic loop). The figure also shows the frequency response of the empty cavity and quartz loaded cavity measured conventionally with the VNA.
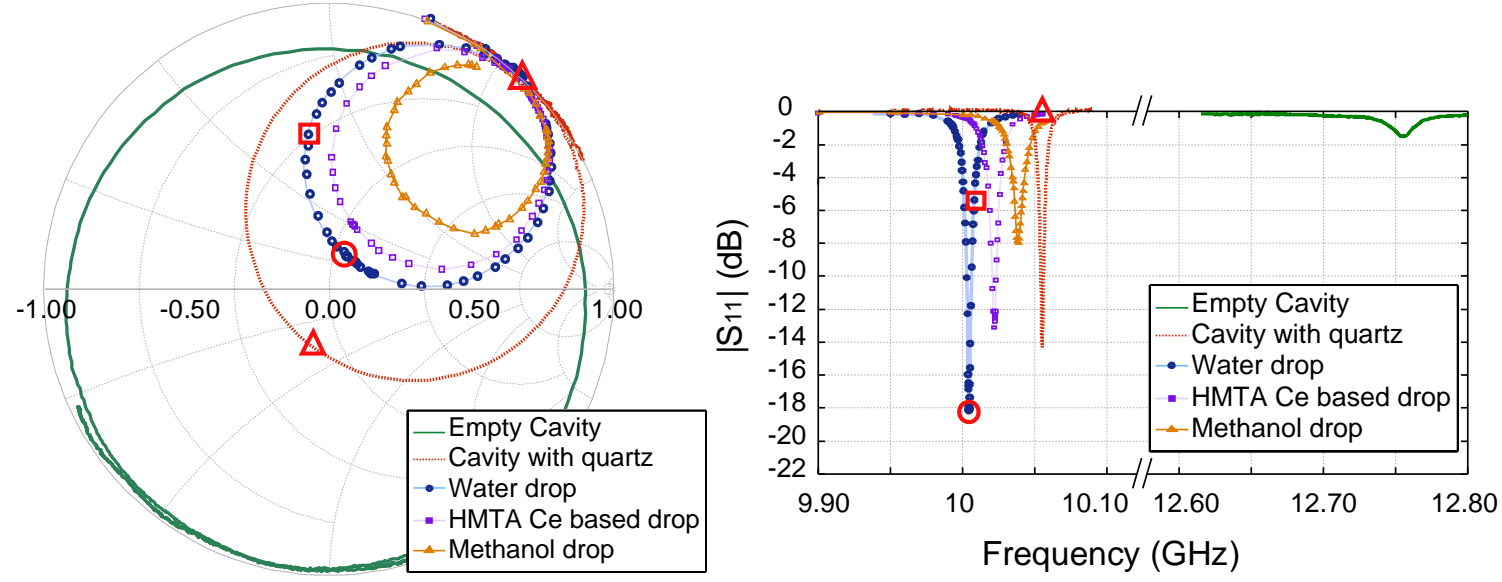

Figure 10. Influence of a drop presence in the center of the cavity on the resonance TM010 mode for different materials. Left: Smith chart representation of the reflexion parameter $S_{11}$. Right: Magnitude of the $S_{11}$. Red marks (circle, square and triangle) correspond to the ones represented in Figure 7. In the case of the Smith chart, the triangle is double represented to indicate the correspondence with the resonance of the cavity with quartz without drop.

Since the representation of the $S_{11}$ parameter around the resonant frequency in this chart is a circle, this complex representation (with real and imaginary part) proved to be very useful to identify the correct module amplitude of each frequency measurement point of the cavity with different drops. It is possible to perceive that the middle points selected in Figure 7 to reconstruct the resonant frequency (red signs) perfectly match over the same single circle in the Smith chart. The red circle mark at $10.005 \mathrm{GHz}$ has the maximal excursion (diameter) in the representation of the $S_{11}$ parameter and guarantees the most suitable resonant frequency value.

Figure 10 (right) represents the frequency response of the magnitude of input return losses $\left(\mathrm{S}_{11}\right)$ of the microwave cavity. Drop measurements are represented together with the measurement of the empty cavity and the cavity with quartz tube, for the resonant mode $\mathrm{TM}_{010}$ in the frequency range (9.9-12.8 GHz). A shift of the resonant frequency for this mode can be seen when a drop is inside the cavity compared to the empty cavity with the quartz tube.

Table 1 summarizes the numerical values of the resonant frequencies $\left(f_{r}\right)$ and $Q$-factors for the measurements illustrated in Figure 10. The calibration parameters of the cavity with the quartz tube, according to the procedure described in [19], were applied to the measurements with drops to determine the unloaded resonant frequencies. It also shows the permittivity values of the samples, obtained with the method described in section 4.1. 
Table 1. Resonant frequencies, $Q$-factors and dielectric properties recovered from the measurements.

\begin{tabular}{|c|c|c|c|c|}
\hline & $f_{r}(\mathrm{GHz})$ & $Q$-factor & $\varepsilon^{\prime}$ & $\varepsilon " / \sigma$ \\
\hline Empty Cavity & 12.7556 & 7532.26 & --- & $2.069 \mathrm{e} 7 \mathrm{~S} / \mathrm{m}$ \\
\hline Cavity with quartz tube & 10.0551 & 6817.01 & 3.67 & 0.0004 \\
\hline $\begin{array}{l}\text { Cavity with quartz and water single drop } \\
\text { ( } 1.47 \text { mm radius) }\end{array}$ & 10.0048 & 1597.05 & $\begin{array}{l}65.63 \pm 3.35 \\
(63.8[20])^{*}\end{array}$ & $\begin{array}{l}29.51 \pm 2.45 \\
(28.7[20])^{*}\end{array}$ \\
\hline $\begin{array}{l}\text { Cavity with quartz and methanol single } \\
\text { drop ( } 1.12 \text { mm radius) }\end{array}$ & 10.0381 & 1038.05 & $\begin{array}{c}10 \pm 0.36 \\
(7.94[21])^{*}\end{array}$ & $\begin{array}{l}8.46 \pm 0.50 \\
(8.22[21])^{*}\end{array}$ \\
\hline $\begin{array}{l}\text { Cavity with quartz and HMTA cerium } \\
\text { based single drop ( } 1.30 \mathrm{~mm} \text { radius) }\end{array}$ & 10.0222 & 1331.77 & $34 \pm 2.83$ & $28.3 \pm 2.56$ \\
\hline
\end{tabular}

* Values measured with coaxial broadband methods for comparison

\subsection{Dielectric Properties}

6 The procedure to determine the permittivity of the drops is based on the Circuit Analysis Method.

7 This method consists of a segmentation of large electromagnetic problems (large structures) into

8 smaller ones. Each simple structure is characterized with the Generalized Admittance Matrix

9 (GAM) and then all the simple structures are joined together, applying the boundary conditions,

10 to analyze the whole complex structure [22-23].

11 Figures 11, 12 and 13 show the dielectric constant and loss factor as a function of the assumed 12 liquid drop radius (fixed by the nozzle of the drop generator). This permittivity values are 13 calculated as a function of the drop radius with the Circuit Method described above, for the 14 resonant frequency and Q-factor of the cavity in each situation (Table I). The relationship can 15 also be used to fix the drop dimensions from a sample of known dielectric properties (such as 16 water) but also indicates how the impact of the uncertainties in the drop dimensions can affect in 17 the permittivity determination.

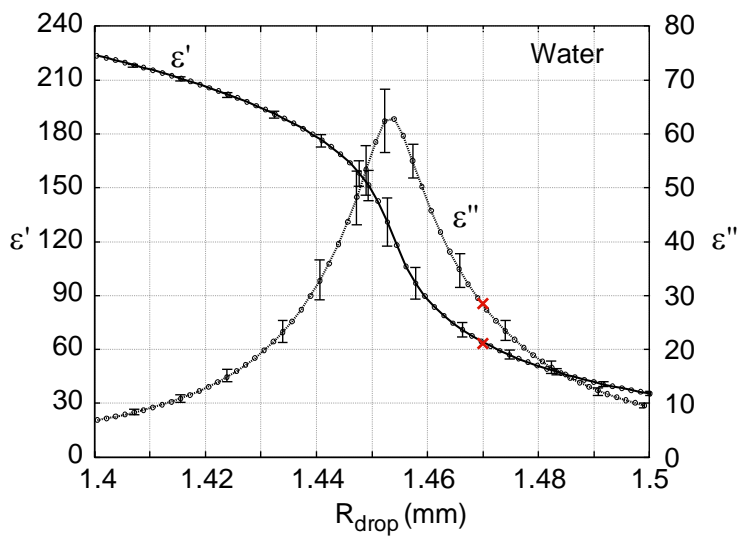

Figure 11. Calculated apparent dielectric properties as a function of assumed water drop radius. 


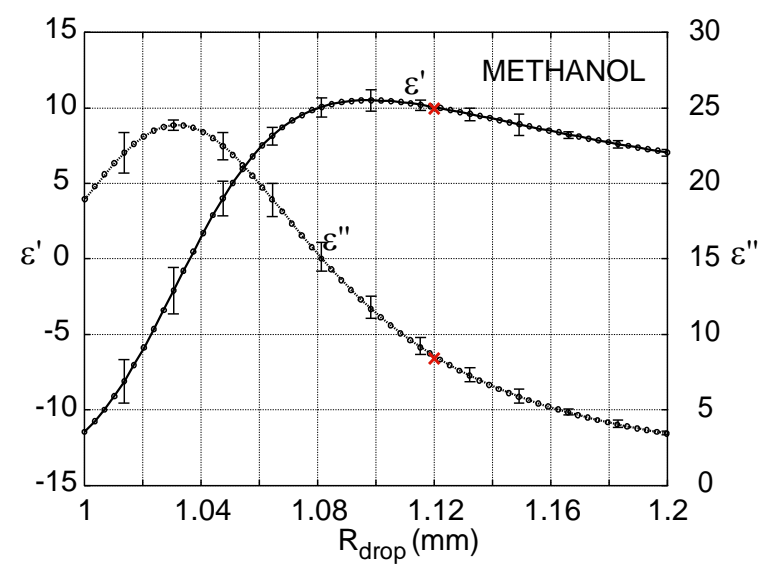

Figure 12. Calculated apparent dielectric properties as a function of assumed methanol drop radius.

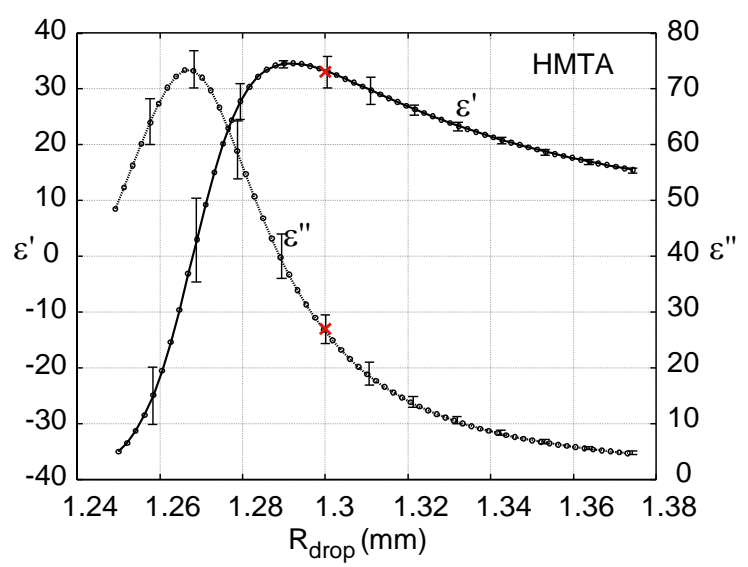

Figure 13. Calculated apparent dielectric properties as a function of assumed HMTA cerium based drop radius.

The uncertainties represented in the Figures 11-13 have been calculated, following the method described in [22, 24, 25], considering a $\pm 0.1 \mathrm{MHz}$ frequency, \pm 5 Q-factor, $\pm 0.1 \mu \mathrm{m}$ cavity radius, $\pm 0.1 \mu \mathrm{m}$ quartz radius and $\pm 1 \mu \mathrm{m}$ cavity height variations. The frequency variation is based on the frequency generator uncertainty, and Q-factor variation has been empirically estimated from the deviation of the Q-factor value after several measurements with the system. Finally, the variations used for the uncertainties of cavity and quartz dimensions are the ones provided by the manufacturers.

Table I shows the values of dielectric properties determined with this procedure for water (1.47 mm radius), methanol (1.12 mm radius) and HMTA cerium based solution $(1.30 \mathrm{~mm}$ radius). In both the case of water and the case of methanol, the calculated results are compared with published values with the open-ended coaxial method [20-21].

As the measured values are in the range of the dielectric properties for water and methanol at these frequencies for the radius given by the drop generator, the method is considered to be valid and allows the measurement of new liquids. Then, the dielectric properties of the HMTA ceriumbased solution used in the MIG process are finally determined.

\section{Conclusion}

A new procedure to measure the dielectric properties of single falling drops has been developed. The procedure is proposed as a non-contact method to meet the need to measure dielectric 
properties in cases such as the radioactive materials proposed in this project. The frequency

2 response of the cavity loaded with a single drop is reconstructed by frequency steps while

3 the drop is falling along the microwave cavity's central axis. Because of the drop dimensions and

4 the technical availability of the experimental development a frequency in the X-band

5 range is used.

6 The dielectric values calculated with the experimental measurements have been satisfactorily

7 validated with previously reported results and allow the use of the procedure with other liquid

8 samples in further work.

9 Moreover, simulations have proved that the experimental measurements can provide information

Table 2. Symbols, Abbreviations and Acronyms

\begin{tabular}{|c|c|c|}
\hline Symbol & Units SI & Parameter \\
\hline MIG & & Microwave Internal Gelation \\
\hline MA & & Minor Actinides \\
\hline $\mathrm{SP}$ & & Sphere Pac \\
\hline$\rho$ & $\mathrm{kg} \cdot \mathrm{m}^{-1}$ & Material density \\
\hline$c_{p}$ & $\mathrm{~J} \cdot \mathrm{kg}^{-1} \cdot \mathrm{K}^{-1}$ & Heat capacity \\
\hline$k_{T}$ & $\mathrm{~W} \cdot \mathrm{m}^{-1} \cdot \mathrm{K}^{-1}$ & Thermal conductivity \\
\hline$h_{T}$ & $\mathrm{~W} \cdot \mathrm{m}^{-2} \cdot \mathrm{K}^{-1}$ & $\begin{array}{l}\text { Convection heat transfer } \\
\text { coefficient }\end{array}$ \\
\hline$T$ & K & Temperature \\
\hline$t$ & s & Time \\
\hline$\varepsilon^{*}$ & $\mathrm{~F} \cdot \mathrm{m}^{-1}$ & Complex permittivity \\
\hline$\varepsilon^{\prime}$ & --- & Dielectric constant \\
\hline$\varepsilon^{\prime \prime}$ & --- & Loss factor \\
\hline$\varepsilon_{0}$ & $\mathrm{~F} \cdot \mathrm{m}^{-1}$ & Vacuum permittivity \\
\hline$Q_{g e n}$ & $\mathrm{~W} \cdot \mathrm{m}^{-3}$ & $\begin{array}{l}\text { Heat generation rate in a } \\
\text { material }\end{array}$ \\
\hline$E_{\text {rms }}$ & $\mathrm{V} \cdot \mathrm{m}^{-1}$ & $\begin{array}{l}\text { Root mean square electric } \\
\text { field }\end{array}$ \\
\hline $\mathrm{S}_{11}$ & $\mathrm{~dB}$ & Input return losses amplitude \\
\hline
\end{tabular}

\section{Nomenclature}

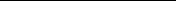

\section{Acknowledgment}

This work is a part of the PINE (Platform for Innovative Nuclear FuEls) project which targets the development of an advanced production method for Sphere-Pac fuel and is financed by the Swiss Competence Center for Energy and Mobility. The work has been also financed by the European Commission through contract no 295664 regarding the FP7 PELGRIMM Project, as well as contract no 295825 regarding the FP7-ASGARD Project. MC-S would like to thank the ITACA research team (UPV Val'encia, Spain) and the EMPA Thun (Switzerland) for their support in the measurements and Carl Beard (PSI, Switzerland) for the help provided in respect with CST simulations. The work of FLP-F was supported by the Conselleria d'Educaci'o of the Generalitat Valenciana for economic support (BEST/2012/010).

\section{References}

[1] M. Salvatores, Nuclear Energy Agency (NEA), "Physics and Safety of Transmutation Systems, A Status Report”, NEA No. 6090, ISBN 92-64-01082-3, OECD Publications, 2006.

[2] M.A. Pouchon, G. Ledergerber, F. Ingold, K. Bakker "Sphere-Pac and VIPAC Fuel” Vol. 3 pp. 275-312 in Comprehensive Nuclear Materials, $1^{\text {st }}$ ed, Ed. Elsevier Ltd, February 2012 (ISBN: 978-0-08-056027-4) 
[3] R. K. McGeary, “Mechanical packing of spherical particles,” J. Am. Ceram. Soc., 44:513-522, 1961.

[4] G. Ledergerber, "Improvements of the Internal Gelation Process," Technical Report, Trans. of the American Nuclear Society, 40, 55-56, 1982.

[5] S. Yamagishi, A. Hasegawa, T. Ogawa, "Development of a Rapid Gelation Apparatus with Microwave Heating," Technical Report 94-010, Japan Atomic Energy Research Institute, 1994.

[6] A. Rosin, A. Schmidt, T. Gerdes, J. Somers, M. Willert-Porada, "Microwave assisted internal gelation of droplets - A case study," Proc. 10th International Conference on Microwave and High Frequency Heating, 346- 349, 2005.

[7] C. Jungo, G. Ledergerber, Process and apparatus for producing microspheres, United States Patent 4,431,164; Japanese Patent 1,548.777, 1984.

[8] G. Ledergerber, "Internal gelation using microwaves," Technical Report, IAEA-TECDOC, Advanced Fuel Technology and Performance (Proc. of An Advisory Group Meeting, Würenlingen, Switzerland, 4-6 Dec. 1984), 352, 165-174, 1985.

[9] M. Cabanes-Sempere, J.M. Catalá-Civera, C. Cozzo, S. Vaucher, M.A. Pouchon, "Innovative production of nuclear fuel by Microwave Internal Gelation: Theoretical microwaves-microspheres interaction,” Proceedings of the 13th International Conference on Microwave and RF Heating, AMPERE 2011, September 5-9, 2011, Toulouse (FRANCE), pp. 231-234. ISBN: 978.2.85428.978.7.

[10] A. Metaxas, R. Meredith, Industrial microwave heating, IEE Power Engineering Series 4, Peter Peregrinus Ltd., London, UK, 1983.

[11] G.Roussy, J. Pearce, Foundations and industrial applications of microwaves and radio frequency fields, John Wiley \& Sons, Inc., 1995.

[12] M. Cabanes-Sempere, J.M. Catalá-Civera, C. Cozzo, S. Vaucher, M.A. Pouchon, "Innovative production of nuclear fuel by Microwave Internal Gelation: Heat transfer model of falling droplets,” Progress in Nuclear Energy, 57 111-116 May 2012.

[13] S.J. Farlow, Partial Differential Equations for Scientists and Engineers, John Wiley \& Sons, Inc. \& Dover Publications, Inc., 1982.

[14] M. Cabanes Sempere, C. Cozzo, J.M. Catalá-Civera, F.L. Peñaranda-Foix, K. Ishizaki, S.Vaucher, M.A. Pouchon, "Characterization of Free Falling Drops inside a Microwave Cavity," Proceedings of the International Microwave Symposium IMS 2012, June 17-22, 2012, Montreal (Canada), pp. 1738-1740. ISBN: 978.1.4673.10868.4. (DOI: 10.1109/MWSYM.2012.6259757)

[15] QWED-QW3D QuickWave Editor and Simulator Reference Guide v.6.5 Warsaw 2006.

[16] C. Cozzo, S. Vaucher, K. Ishizaki, D. Megias-Alguacil, M.A. Pouchon, "Chemistry of uranium surrogate during microwave assisted internal gelation for fuel fabrication,” Proc. of GLOBAL 2011, December 11-16, 2011, Makuhari (JAPAN), paper No. 392501.

[17] Y. W. Lee, H. S. Kim, C.Y. Joung, S.H. Na, G. Ledergerber, P. Heimgartner, M.A. Pouchon, M. Burghartz, "Preparation of simulated inert matrix fuel with different powders by dry milling method," J. Nucl. Mater., 274:714, 1999.

[18] CST Microwave Studio, Workflow \& Solver Overview. Computer simulation technology AG, CST 1998-2012.

[19] A. J. Canós, J.M. Catalá-Civera, F.L. Peñaranda-Foix, E. de los Reyes-Davó “A Novel Technique for Deembeding the Unloaded Resonance Frequency From Measurements of Microwave Cavities,” IEEE Trans. Microwave Theory \& Tech., vol. 54, no. 8, pp. 3407-3416, August 2006.

[20] U. Kaatze, “Complex Permittivity of Water as a Function of Frequency and Temperature,” J. Chem. Eng. Data., vol. 34 No. 4 pp. 371-374, 1989.

[21] U. Kaatze, "Reference liquids for the calibration of dielectric sensors and measurement instruments," Meas. Sci. Technol., vol. 18 pp. 967-976, 2007.

[22] Felipe L. Penaranda-Foix and Jose M. Catala-Civera, "Circuital analysis of cylindrical structures applied to the electromagnetic resolution of resonant cavities”, Chapter 7 in Passive Microwave Components and Antennas, 1st ed, Ed. IN-TECH, April 2010 (ISBN 978-953-307-083-4).

[23] Felipe L. Peñaranda-Foix, Michael D. Janezic, Jose M. Catala-Civera and Antoni J. Canos, Full-Wave Analysis of Dielectric-Loaded Cylindrical Waveguides and Cavities Using a New Four-Port Ring Network. IEEE Trans. Microwave Theory \& Tech., vol. 60, no. 9, pp. 2730-2740, September 2012.

[24] Baker-Jarvis J, Janezic M D, Domich P D and Geyer R G 1994, Analysis of an open-ended coaxial probe with liftoff for nondestructive testing IEEE Trans. Instrum. Meas. 43 711-8

[25] Bell S 1999 Beginner's Guide to Uncertainty of Measurement Good Practice Guide No. 11 (Teddington: National Physical Laboratory) 